\title{
Building integrated photovoltaic design of nonlinear airport roof based on ruled surface fitting
}

\author{
Jinting Sui ${ }^{1}$, Longwei Zhang ${ }^{1}$ * \\ ${ }^{1}$ School of Architecture and Urban Design, Shenyang Jianzhu University, 110168 Shenyang, China
}

\begin{abstract}
Solar energy is a new renewable energy. Using solar energy to generate electricity is an effective means to achieve sustainable development. The huge roof at the top of the airport is a natural interface to receive solar energy, which can be used to supply the energy needed by the airport through building integrated photovoltaic design. However, due to the curved shape of the airport roof, there are limitations in solar energy acquisition and construction of the airport roof. In this paper, the method of "ruled surface fitting-solar radiation analysis-modular unit arrangement" is used to optimize the shape of the non-linear airport roof, and convert the complex roof surface shape into a straight line with good sunlight performance and easy construction. The ruled surface can better integrate BIPV and nonlinear airport, and improve the solar power generation efficiency of the building, thus improving the sustainable performance of the building.
\end{abstract}

\section{Background}

With the rapid development of economy, the concept of sustainable development has become increasingly popular in the world. Many countries regard solar energy as an important emerging industry. The scale of global solar energy development and utilization is rapidly expanding, technology keeps improving and cost is significantly reduced, showing a good development prospect. The introduction of building integrated photovoltaic is not only in line with China's strategic planning for sustainable development of energy in the 21 st century, but also an important part of developing a circular economic model and the construction of a harmonious society. this is also of great significance to promote the use of solar energy and the development process of the photovoltaic power generation industry.

By the end of June 2020, the number of registered airports in China has reached 296, and the number of airports in China is growing exponentially. With the rapid growth of airport construction, the problem of high energy consumption is becoming more and more prominent. In order to meet the airport energy demand without damaging the ecological environment, the use of solar power generation, especially the large-scale development and application of BIPV is particularly important. The huge building volume and large roof area of the airport are favorable conditions to apply the buildings integration photovoltaic in the airport. First of all, the airport roof area is large, the shape is usually gentle and complete, and the complexity of the form is low, which meets the requirements of photovoltaic integration for uniform and smooth interface receiving light. Secondly, the airport covers a large area, has a low building density in the surrounding environment and an open site. Therefore, the solar radiation receiving surface is less blocked by adjacent buildings, which can effectively improve the irradiation time and irradiation quality of the roof exposed to light interface. Therefore, as to a civil transport airport with vast roof and huge annual energy consumption, it can effectively alleviate the energy consumption pressure of the airport by integrating it with photovoltaic buildings.

This research takes "non-linear airport roof photovoltaic integration" as the research direction, applies photovoltaic integration technology on the airport roof, constructs a new type of nonlinear airport photovoltaic roof system, and further summarizes a set of airport roof BIPV implementation method, which allows the nonlinear roof shape to be organically combined with the planar photovoltaic unit to solve the problem of combining BIPV with non-linear airports. This article takes the corridor type of typical airport roof as an example, and elaborates the design method of airport photovoltaic roof in detail.

\section{Topology optimization of macroscopic shape}

Based on the nonlinear airport roof shape, this paper introduces the ruled surface architecture logic under the premise of meeting its form and function requirements. By adding wires and bus bars, the nonlinear airport roof is transformed into a ruled surface, so that the nonlinear shape is constructed by geometrical logic construction, which lays the foundation for the later arrangement of photovoltaic panels. Secondly, using the solar radiation analysis software Ladybug and the multi-objective genetic algorithm tool Octopus, the topological optimization of

\footnotetext{
${ }^{*}$ Corresponding author: z lw@sjzu.edu.cn
} 
the shape is carried out on the basis of solar radiation, prototype fit, and construction implementation degree [1], and the optimal solution is selected.

\subsection{Parameterized geometric logic construction}

Most of the non-linear airport roof are free-form surfaces, which are complex, diverse and irregular. Direct application of photovoltaic panels cannot fit free-form surfaces. Therefore, the primary basis for the combination of the two is to introduce geometric logic to reconstruct the nonlinear airport roof, and use ruled surface as the transition between the two. The ruled surface is composed of wires and straight bus bars. In the wire dimension, it is a free curve to control the straight lines. The spatial shape and contour of the curved surface ensure the shape of the free-form surface. The dimension of the bus bar is a straight line with certain regularity. The arrangement method controls the shape of the free-form surface to ensure the reasonable fitting of the photovoltaic unit.(Figure 1).

Taking the typical form of airport roof as an example, the integration process of nonlinear airport roof and photovoltaic building integration is further illustrated as follows. Firstly, based on the form and function of the nonlinear airport roof, the nonlinear morphological features are analyzed, and the upper and lower boundary lines are extracted as the wires of the ruled surface. Secondly, the planned copper indium gallium selenide photovoltaic module size $600 * 1200$ is set as the unit modulus. Based on the grasshopper platform, combined with the airport structure, it is divided horizontally with $600 \mathrm{~mm}$ as the modulus along the wires through the vertical generatrix, and $1200 \mathrm{~mm}$ as the modulus for vertical division. Then the free-form surface is divided into several $600 * 1200$ units according to geometric logic. The horizontal and vertical division transforms the initial nonlinear surface into a ruled surface with geometric relations, generates a geometric model with parameterized logic, and transforms the complex and irregular free-form surface into a relatively simple and easy-to-control dynamic model [2].

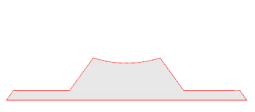

Guide line extract

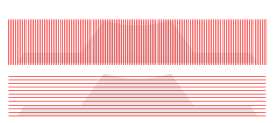

Generatrix extract

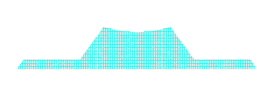

Ruled surface
Figure 1. Geometric logic construction of ruled surface.

\subsection{Solar radiation analysis}

The analysis of sunlight acquisition is the premise and foundation of the integration of solar photovoltaic application. Under the premise of ensuring the nonlinear form of the building, the main goal of the combination of BIPV and airport roof is to achieve the maximum solar radiation acquisition. The LadyBug tool based on Grasshopper platform is used to simulate the solar radiation amount of roof [3] and generate the distribution map of annual sunshine access, which provides data support for roof shape optimization to obtain the maximum solar radiation amount [4].

In this essay, the genetic algorithm tool Octopus is used to generate a large number of alternative roof forms with the annual solar radiation as the target, and automatic screening is conducted to obtain three optimal surfaces (Figure 2). On this basis, solar radiation amount, prototype fit degree, construction implementation degree and space comfort degree are taken as the criteria to compare the three:

Scheme 1: The annual solar radiation of this form is $1,753.91 \mathrm{kWh} / \mathrm{m}^{2}$. Although the solar radiation obtained per unit area is relatively high, the agreement between this surface and the initial form of the airport roof is relatively low. In addition, due to the large protrusion on the upper part, the internal space of the terminal building is wasted, and the space utilization rate is low. Meanwhile, the local protrusion is too large, and the continuity of the curved surface is not strong.

Scheme 2: It has a high fit with the original form of the airport, but the annual solar radiation of this form is $1688.49 \mathrm{kWh} / \mathrm{m}^{2}$, and the amount of sunshine obtained is lower than that of other schemes.

Scheme 3: The annual solar radiation of this body is $1691.02 \mathrm{kWh} / \mathrm{m}^{2}$, the annual solar radiation acquisition is high, and the initial form of the airport roof has a high fit degree, the curved surface has strong continuity, and the space utilization rate is high.

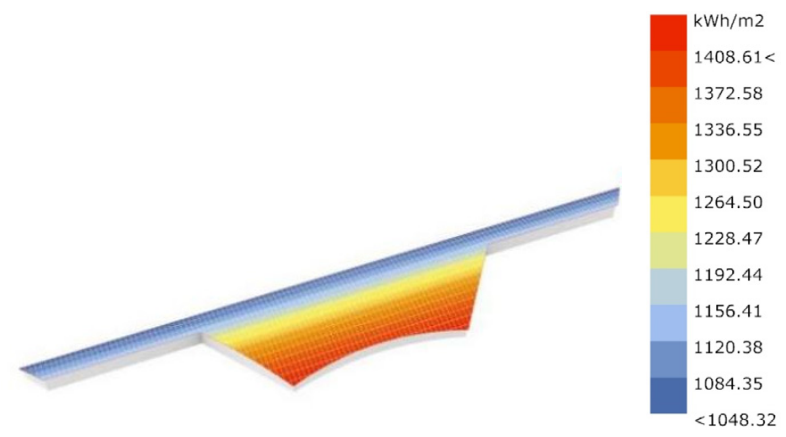

Scheme1 Roof axonometric

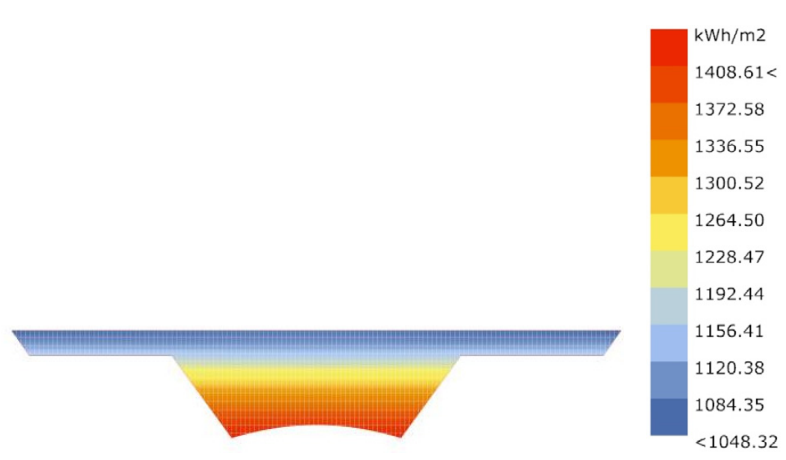

Scheme1 Solar radiation data 


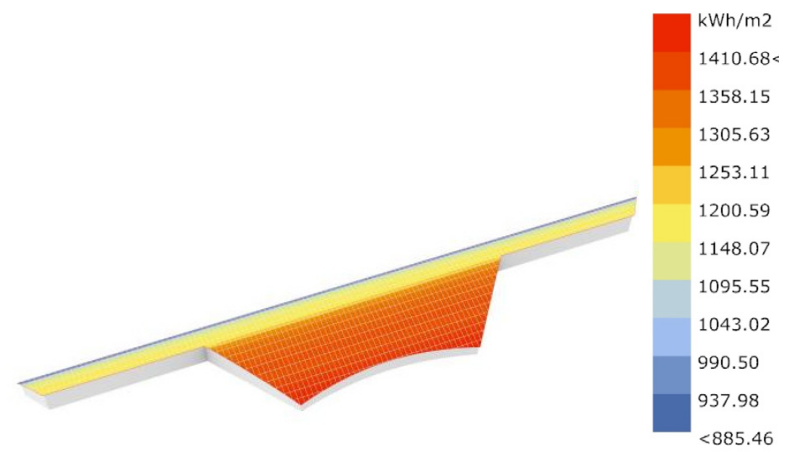

Scheme2 Roof axonometric

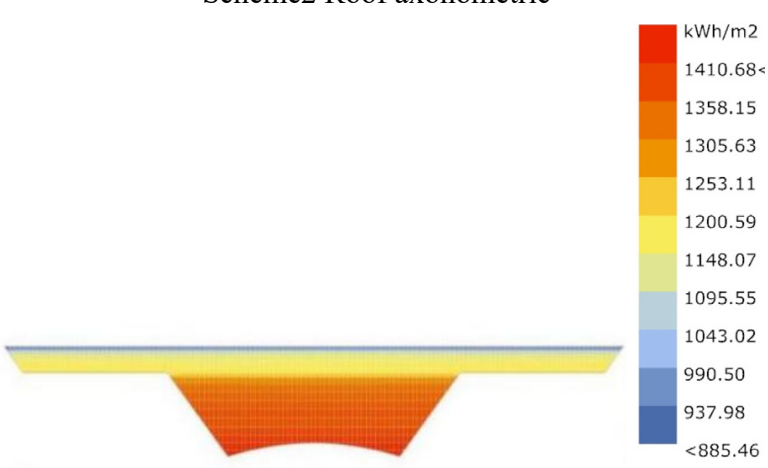

Scheme2 Solar radiation data

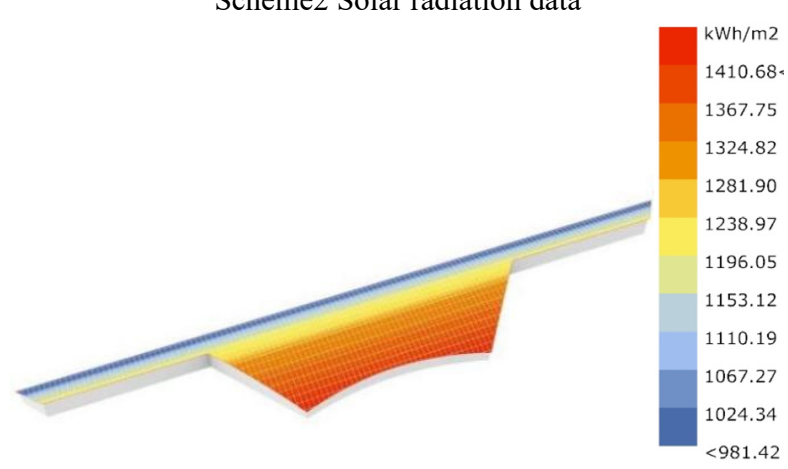

Scheme3 Roof axonometric

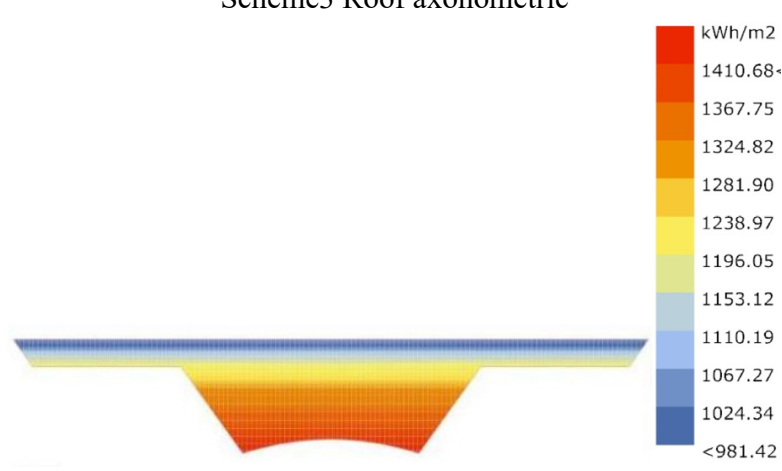

Scheme3 Solar radiation data

Figure 2. Typical optimization scheme and solar radiation data

The analysis shows that the scheme has high solar radiation acquisition amount, good morphology fit, full sunlight receiving area and the best comprehensive performance in the three years. It has a good optimization space and is suitable for the foundation of nonlinear airport roof.

\section{Mesoscopic shape surface partition}

Based on Grasshopper platform, interval processing was carried out on the sunshine analysis data, gradient partition was carried out according to the solar irradiance, and regions were divided on the roof surface [5], which were divided into areas with sufficient sunshine, intermittent sunshine and insufficient sunshine. In order to meet the basic shape and function requirements of the roof, the form of the intermittent sunshine area was partially adjusted to convert it into a full sunshine area, and the laying area of photovoltaic panels was increased.

\subsection{Airport roof surface partition}

On the one hand, the surface partition of the airport roof determines the functional partition according to the structure and function. According to the function of the corridor type airport, it is divided into three areas, namely, two corridor areas and one terminal area. On the other hand, the photovoltaic installation area is determined through annual solar radiation analysis. According to the sunshine analysis data above, the annual solar radiation acquisition can be roughly divided into three gradients. The red gradient is the area with sufficient sunshine, and the annual solar radiation acquisition is greater than $1000 \mathrm{kWh} / \mathrm{m}^{2}$. Yellow gradient for intermittent sunshine area, annual solar radiation acquisition in $800-1000 \mathrm{kWh} /$ $\mathrm{m}^{2}$; The blue gradient is the insolation lack area, the annual solar radiation acquisition amount is less than $800 \mathrm{kWh} /$ $\mathrm{m}^{2}$; The straight grain surface is divided into three regions to form three straight grain surfaces. The rationalized airport roof surface partition is generated from the above two aspects(Figure 3).

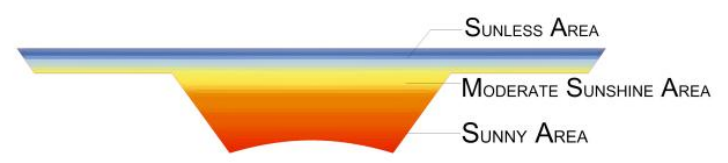

Solar radiation partition

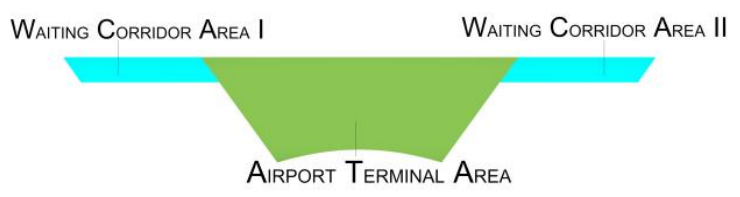

Function partition

Figure 3. Surface partition.

\subsection{Shape partition optimization}

The red gradient does not need to be adjusted due to high solar radiation. The blue gradient solar radiation is too low, which can only be satisfied if the body changes greatly, and the adjustment meaning is low. On the basis of the local adjustment of the shape, the yellow gradient can be transformed into a full sunshine area, which has great adjustment value. In this paper, genetic algorithm is used to make local adjustment of the surface of intermittent sunshine zone with the objective of maximizing the annual 
solar radiation acquisition [6]. At the same time, using Kangaroo tool of Grasshopper platform, the intermittent sunshine area surface edge is fixed, to ensure that the body and the full sunshine area and the lack of sunshine smooth connection. Through dynamic feedback model real-time adjustment, the intermittent sunshine area is effectively converted into a full sunshine area, thus improving the overall body's annual solar radiation acquisition. In the process, the bucket effect of photovoltaic should be paid attention to, which refers to the photovoltaic power generation system in general with 6-9 photovoltaic cell to form a set of photovoltaic (pv) set of strings, and the group list of photovoltaic cell within the power generation efficiency should be close. Otherwise, similar to the barrel theory, the whole power generation efficiency is determined by photovoltaic power generation efficiency with the lowest unit, thus forming a greater influence of the uniformity on the photovoltaic efficiency. Based on the above analysis, two variables, annual solar radiation amount and body uniformity, should be taken as optimization objectives when adjusting the body locally, so as to effectively improve the efficiency of photovoltaic power generation.

\section{Microscopic shape modularization}

Optimized by the above steps, the roof shape is transformed into two large areas -- a sunny area and a lack of sunshine area. Firstly, photovoltaic units are introduced in the area with sufficient sunshine. Based on Grasshopper platform, photovoltaic units are taken as modules to modularize the surface of the body. The sunshine deficiency area is laid with ordinary material units. Secondly, genetic algorithm tools were used to optimize the sunlight incidence angle of each photovoltaic module [7] according to the sun angle to form a scale-type roof arrangement. The arrangement of modules reflects the geometric construction logic of the overall shape of the roof, and reduces the difficulty of construction.

\subsection{Standardized module unit}

Standardization of module units plays a crucial role in the construction and implementation of the scheme. If the body fit degree is completely pursued, the size of each photovoltaic unit is different, which greatly increases the construction period and difficulty. Therefore, standardization of module units is needed. In the shape topology optimization stage, although the initial horizontal and vertical partitioning was carried out through the $600 * 1200 \mathrm{~mm}$ size of photovoltaic cell, the size changed during the fitting process. Therefore, the Kangaroo tool of Grasshopper platform is used to introduce dynamics calculation into $\mathrm{GH}$ and achieve interactive simulation, form-finding optimization, constraint solution through physical and mechanical simulation. The tool controls four factors: edge, side length, control point and rotation angle, and standardizes it to $600 * 1200 \mathrm{~mm}$ by using the principle of elasticity. After optimization, although the size of each unit fluctuates slightly, the value is within the allowable range of construction error, reaching the standard module unit(Figure 4).

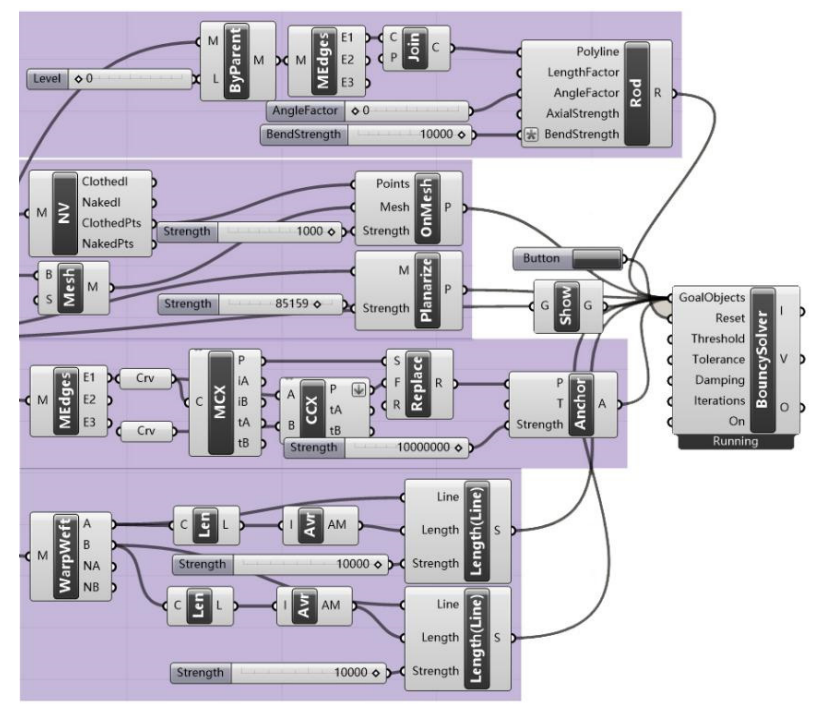

Figure 4. Standardized unit program.

\subsection{Photovoltaic cell detail optimization}

After the fitting of the photovoltaic cells and the nonlinear airport roof, the shading between the photovoltaic cells and the rotation angle of the photovoltaic panels have a crucial effect on the efficiency of photovoltaic power generation. In order to achieve the maximum solar radiation acquisition, each photovoltaic unit needs to rotate at a certain angle to receive sunshine. At the same time, in order to avoid the shadow blocking between photovoltaic panels affecting the efficiency of photovoltaic power generation, a shadow transition zone is introduced between photovoltaic panels. Under the premise of keeping the large structure of the roof shape unchanged, the Octopus tool was used for multi-objective optimization. The optimization variables are the rotation angle and the width of the shadow transition band. Considering the uniformity and practicability of the roof shape, the rotation Angle range of each photovoltaic module is controlled within $\pm 5^{\circ}$ compared with the original surface, and the width of the shadow transition band is controlled within $100 \mathrm{~mm}$. By adjusting the rotation angle of the photovoltaic unit and the width of the shadow transition zone, the material consumption is minimized on the premise of guaranteeing the annual solar radiation acquisition, and the economic efficiency and realizability of construction are increased. After several iterations through the Octopus tool, the final optimal shape of the airport roof is formed, and the organic combination of photovoltaic integration and nonlinear airport roof is realized(Figure 5). 


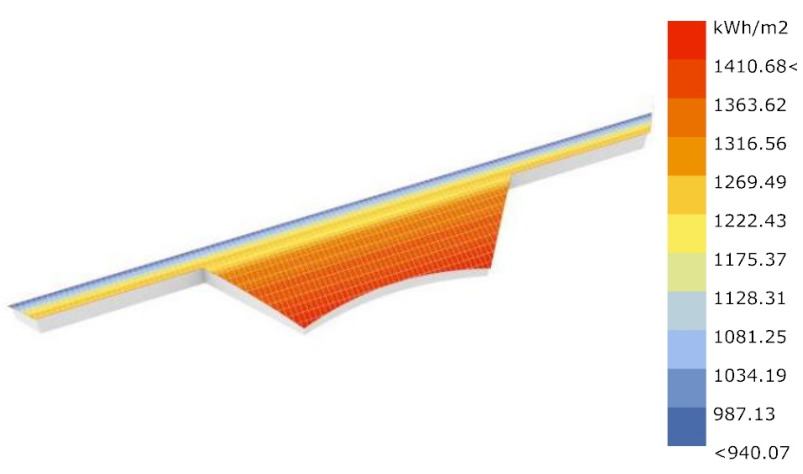

Roof axonometric

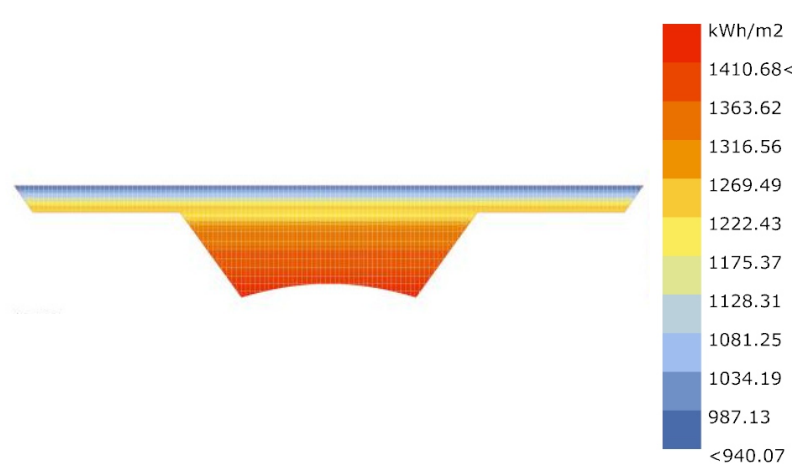

Solar radiation data

Figure 5. Final optimization scheme.

\section{Summary}

This essay proposes a nonlinear airport roof optimization method based on ruled surface fitting methods, which can transform complex and irregular roof free-form surfaces into ruled surfaces with good sunlight performance and easy construction, making better integration of BIPV and nonlinear airport.

(1)Establish geometric logic, and implement the horizontal and vertical fitting of the airport roof by introducing a $600 * 1200$ modulus to realize the preliminary standardization of the roof shape. At the same time, the dynamic parameter frame is used to realize the data linkage from the whole to the part of the roof shape, which lays the foundation for the optimization of the genetic algorithm based on the grasshopper platform, and realizes the shape-level fitting of the nonlinear roof shape.

(2) The sunshine data of the airport roof shape is analyzed by the Ladybug tool, and by gradient partitioning, it is divided into sunshine sufficient area, sunshine intermittent area and sunshine insufficient area, which provides quantitative basis for further optimizing the shape. Through zone fitting and local adjustment of the shapes of intermittent sunshine areas and low sunshine areas, group-level fitting of nonlinear roof shapes is realized, which effectively increases the photovoltaic paving area and improves the efficiency of photovoltaic power generation.

(3)The standardization of photovoltaic module units is an important factor affecting the feasibility of construction. Through the Kangaroo tool, the principle of elasticity is used to standardize it, and at the same time, based on the Octopus tool, with the annual solar radiation acquisition as the optimization goal, the optimal rotation angle and transition zone width of the photovoltaic unit are obtained. From macro to micro, it realizes the structural-level fitting of nonlinear roof shapes, and transforms complex freeform surfaces into a highly feasible nonlinear integrated photovoltaic airport.

Non-linear airport roofs provide us with a new way of efficiently using solar energy from the perspective of renewable energy utilization. Because of its huge solar receiving surface, it has a huge advantage in the application of photovoltaic power generation. Whether it is the reconstruction of existing airports or the construction of new airports, it is easy to form a cluster effect, and does not occupy land, which greatly reduces the investment cost of photovoltaic facilities and alleviates the problem of high energy consumption in the airport. It has a large application prospect and development potential, and complies with China's Energy Strategic Plan for Sustainable Development in the 21st Century.

\section{Acknowledgments}

The authors gratefully acknowledge the support of National Natural Science Foundation of China (Grant 51978418，51738006) and Scientific Research Plan of the Educational Department of Liaoning Province(Grant lnjc202003).

\section{References}

1. J. Harding, S. Joyce, P. Shepherd, C. Williams, Thinking topologically at early stage parametric design, in: Adv. Archit. Geom., Springer, Vienna, 2012, pp. 67-76.

2. Negendahl, Building performance simulation in the early design stage: an introduction to integrated dynamic models, Autom. Constr. 54 (2015) 39-53.

3. Qingsong M, Fukuda H. Parametric Office Building for Daylight and Energy Analysis in the Early Design Stages. Procedia - Soc Behav Sci 2016;216:818-28.

4. Longwei Zhang. Lingling Zhang, Yuetao Wang, Shape optimization of free-form buildings based on solar radiation gain and space efficiency using a multi-objective genetic algorithm in the severe cold zones of China, Solar Energy 132 (2016) 38-50.

5. Y.G. Yi, A.M. Malkawi., 2009. Optimizing building form for energy performance based on hierarchical geometry relation, Automation in Construction. 18. 825-833.

6. Jin, J.T., Jeong, J.W., 2014. Optimization of a freeform building shape to minimize external thermal load using genetic algorithm. Energy Build. 85, 473482.

7. Kämpf, J.H., Montavon, M., Bunyesc, J., Bolliger, R., Robinson, D., 2010. Optimisation of building's solar irradiation availability. Sol. Energy 84, 596-603. 